\title{
Prevalence of Non-alcoholic Fatty Liver Disease in the Russian Federation: the Open, Multicenter, Prospective Study, DIREG 1
}

\author{
Oxana Drapkina*, Yulia Evsyutina, Vladimir Ivashkin \\ Department of Internal Disease Propaedeutics, Gastroenterology and Hepatology, Sechenov First Moscow State Medical University, \\ Moscow, Russian Federation \\ *Corresponding author: drapkina@bk.ru
}

Received April 07, 2015; Revised May 19, 2015; Accepted May 29, 2015

\begin{abstract}
Background: Non-alcoholic fatty liver disease (NAFLD) is a very serious medical and social problem that can lead to the progression of liver cirrhosis and eventually hepatic failure. There is a paucity of data regarding the prevalence and risk factors for NAFLD in the Russian Federation. Methods: The open multicenter prospective screening study, DIREG 1, involved patients aged 18-80 years who were admitted to various polyclinics for ambulatory therapeutic care (with or without apparent signs of hepatic diseases) at 208 centers in the Russian Federation from March 25th to November 26th, 2007. NAFLD was diagnosed by ultrasonography; a range of clinical and laboratory findings were also recorded for each participant. Results: A total of 30,754 outpatients were included in the screening study analysis. The prevalence of NAFLD was $27.0 \%(8,315 / 30,754)$, of which $80.3 \%$ had steatosis, $16.8 \%$ had steatohepatitis and $2.9 \%$ had disease at the cirrhotic stage. Most cases of NAFLD were observed in those aged 50-59 years (31.1\%), 40-49 years (23.6\%), and 60-69 years (18.1\%). The most common risk factors associated with the NAFLD cohort vs the general screening population were dyslipidemia ( $75.9 \%$ vs $37.6 \%)$, arterial hypertension (69.9\% vs $41.8 \%$ ), and hypercholesterolemia (68.8\% vs $33.5 \%$ ) (all $\mathrm{P}<0.001$ ). Conclusion: There is an urgent need to address the high prevalence of NAFLD in the Russian Federation (27\%) and the large proportion of individuals with associated risk factors.
\end{abstract}

Keywords: non-alcoholic steatohepatitis, steatosis, cirrhosis, obesity, dyslipidemia

Cite This Article: Oxana Drapkina, Yulia Evsyutina, and Vladimir Ivashkin, "Prevalence of Non-alcoholic Fatty Liver Disease in the Russian Federation: the Open, Multicenter, Prospective Study, DIREG 1.” American Journal of Clinical Medicine Research, vol. 3, no. 2 (2015): 31-36. doi: 10.12691/ajcmr-3-2-3.

\section{Introduction}

Non-alcoholic fatty liver disease (NAFLD) involves fatty infiltration (steatosis) of the liver, and is diagnosed by various visual or histological examinations, in the absence of alcohol consumption or use of steatogenic medication or hereditary disorders. [1] NAFLD encompasses a broad spectrum of liver disease from benign steatosis to steatohepatitis (NASH) to progressive fibrosis and liver cirrhosis. [2] Long-term studies have shown that NASH increases overall mortality by $35-85 \%$ compared with the age and sex-matched general population. [3] Furthermore, liver-related mortality is increased 9-10-fold with cirrhosis. As with other liver diseases that cause cirrhosis, NAFLD increases the risk of hepatocellular carcinoma, a disease with poor outcomes and limited therapeutic options. [4] In addition to liver morbidity and mortality, NAFLD also increases the risk of cardiovascular disease and type 2 diabetes. [3,5,6] NAFLD can also impose substantial costs to the patient and the health care system. [7]
The prevalence of NAFLD is rising and it is now recognized as the most common chronic liver disease in many parts of the world. [3,8,9] According to previous studies, the prevalence of NAFLD ranges from $6 \%$ to $40 \%$ based on a variety of assessment methods, whereas the prevalence of NASH ranges from $3 \%$ to $9 \%$. $[1,10,11]$ Established risk factors for NAFLD include obesity, type 2 diabetes mellitus, dyslipidemia and metabolic syndrome. $[1,6,12,13]$.

However, other factors are now known to have associations with NAFLD including polycystic ovarian syndrome, hypothyroidism, obstructive sleep apnea, hypopituitarism, hypogonadism, and pancreato-duodenal resection. $[1,14,15]$ More studies are needed to better understand the incidence of NAFLD and its risk factors across different age, ethnic, and geographic groups. The aim of this investigation was to further assess the epidemiology of NAFLD by evaluating its prevalence and risk factors in a large, nationwide population study conducted in the Russian Federation.

\section{Materials and Methods}




\subsection{Patients}

The open multicenter prospective screening study, DIREG 1, was conducted at 208 medical centers in the Russian Federation from March 25th, 2007 to November 26th, 2007. Consecutive patients aged 18 to 80 years admitted to various polyclinics for ambulatory therapeutic care regarding many diseases, with or without apparent signs of hepatic diseases, were included. Exclusion criteria were as follows: refusal to participate in the study, pregnancy and participation in other clinical studies (at this time or in the course of the 3 recent months).

The study was conducted in accordance with the latest version of the World Medical Association Declaration of Helsinki, the European Guidelines for the Rules of Conduct of Clinical Studies, International Conference of Harmonisation (ICH) Guidelines for Conduct of Clinical Studies, and current Russian legislation. A written informed consent was received from all patients before their inclusion in the program.

\subsection{Study Design}

All patients included in the study were subject to history taking and physical examination. Laboratory tests included serum alanine aminotransferase (ALT), aspartate aminotransferase (AST), gamma-glutamyl transpeptidase (GGT), prothrombin time, fasting blood sugar, cholesterol, triglycerides, thrombocytes, hepatitis B surface antigen (HBsAg) and hepatitis C virus (HCV) antibodies. All patients were subject to ultrasonography for measurement of liver, pancreas and spleen size, and the diameter of the portal vein and the splenic vein; for assessment of the structure of the liver and the presence of steatosis, fibrosis and cirrhosis; for assessment of the structure of the pancreas and the presence of steatosis and fibrosis, and for identifying signs of portal hypertension. NAFLD was diagnosed based on ultrasound findings; hepatic biopsy was not carried out due to the large patient numbers involved.

The primary objective was to evaluate NAFLD incidence in the included outpatients. Other important endpoints were the different types of NAFLD e.g. cirrhotic and non-cirrhotic stages, and steatosis and steatohepatitis in patients with an approved diagnosis of NAFLD and by age group. In addition, the prevalence of NAFLD risk factors was evaluated.

\subsection{Statistical Analysis}

Results of summary outcome measures were reported as mean (SD) and proportions. All statistical tests were conducted with the use of two-sided hypothesis with significance value of $<0.05$.

\section{Results}

After applying our exclusion criteria, 30,787 primarycare participants were available for analysis. Subsequently, 33 patients were excluded from the analysis due to lack of a full dataset, and the final analysis included 30,754 subjects.

\subsection{Clinical Characteristics of the Study Population}

Of the total population, 56\% were female and the mean (SD) age was $47.8 \pm 16.4$ years. Patients were categorized by age into 6 groups: 18-29 years (16.8\%), 30-39 years (16.5\%), 40-49 years (21.2\%), 50-59 years (21.0\%), 60-69 years $(12.4 \%)$ and $70-80$ years (8.8\%) (Table 1$)$. Obesity (according to body mass index [BMI]) was recorded in 7,328 patients: $15.7 \%$ had stage 1 obesity, $4.1 \%$ had stage 2 obesity and $2.1 \%$ had stage 3 obesity. The study population commonly had arterial hypertension (41.8\%) or dyslipidemia (32.4\%), with $9.0 \%$ having type 2 diabetes mellitus.

Table 1. Characteristics of the study population

\begin{tabular}{|l|l|}
\hline \multicolumn{2}{|c|}{ Age (years) } \\
\hline \multicolumn{2}{|c|}{ Number of participants (\% of total) } \\
\hline $18-29$ & $5,158(16.8)$ \\
\hline $40-39$ & $5,062(16.5)$ \\
\hline $50-59$ & $6,520(21.2)$ \\
\hline $60-69$ & $6,469(21.0)$ \\
\hline $70-80$ & $3,825(12.4)$ \\
\hline Obesity & $2,693(8.8)$ \\
\hline Nil & \\
\hline Stage 1 & $17,975(58.4)$ \\
\hline Stage 2 & $4,827(15.7)$ \\
\hline Stage 3 & $1,869(4.1)$ \\
\hline \multicolumn{2}{|c|}{ Coexistent diseases } \\
\hline Arterial hypertension & $12,864(41.8)$ \\
\hline Dyslipidemia & $9,967(32.4)$ \\
\hline Type 2 diabetes mellitus & $2,755(9.0)$ \\
\hline
\end{tabular}

\subsection{Ultrasound Characteristics and Laboratory Findings of the Study Population}

In total, $16.3 \%$ of the study population had an enlarged liver based on ultrasound findings, and $24.7 \%$ had heterogeneous structures within the liver (Table 2). Liver steatosis was observed in $24.2 \%$ of subjects, with fibrosis and cirrhosis seen in $2.3 \%$ and $0.8 \%$, respectively. In total, $0.22 \%$ of patients had hepatocellular carcinoma.

Table 2. Ultrasound characteristics of liver, pancreas, and spleen among the study population

\begin{tabular}{|l|l|l|}
\hline \multicolumn{3}{|l|}{} \\
\multicolumn{1}{|l|}{ of total) } \\
\hline & Yes & No \\
\hline Enlarged liver & $5,017(16.3)$ & $20,482(66.6)$ \\
\hline Heterogeneous structure of the liver & $7,590(24.7)$ & $16,788(54.6)$ \\
\hline Increased diameter of the portal vein & $398(1.3)$ & $22,044(72.9)$ \\
\hline Enlarged pancreas & $998(3.2)$ & $22,426(2.1)$ \\
\hline Disturbed structure of the pancreas & $4,363(14.2)$ & $19,209(62.5)$ \\
\hline Enlarged spleen & $387(1.3)$ & $22,853(74.3)$ \\
\hline Increased diameter of the splenic vein & $392(1.3)$ & $21,804(70.9)$ \\
\hline Liver steatosis & $7,456(24.2)$ & $14,492(47.1)$ \\
\hline Liver fibrosis & $692(2.3)$ & $19,877(64.6)$ \\
\hline Liver cirrhosis & $243(0.8)$ & $20,308(66.0)$ \\
\hline Signs of pancreatic steatosis & $622(2.0)$ & $19,901(64.7)$ \\
\hline Signs of pancreatic fibrosis & $2,983(9.7)$ & $17,741(57.7)$ \\
\hline Signs of portal hypertension & $288(0.9)$ & $20,147(65.5)$ \\
\hline
\end{tabular}

Normal levels of ALT, AST and GGT were reported in approximately $70 \%$ of subjects in the study population (Table 3). Elevated levels of ALT $\geq 1.5$ times the upper 
limit of normal (ULN) were observed in 3.3\% of the study population, while $2.6 \%$ had AST $\geq 1.5 \times$ ULN.

Table 3. Laboratory findings among the study population

\begin{tabular}{|c|c|}
\hline & Number of participants (\% of total) \\
\hline \multicolumn{2}{|l|}{ ALT } \\
\hline$<1.5 \mathrm{~N}$ & $3,144(10.2)$ \\
\hline$\geq 1.5 \mathrm{~N}$ & $1,022(3.3)$ \\
\hline Normal & 21,452 (69.8) \\
\hline \multicolumn{2}{|l|}{ AST } \\
\hline$<1.5 \mathrm{~N}$ & $2816(9.2)$ \\
\hline$\geq 1.5 \mathrm{~N}$ & $802(2.6)$ \\
\hline Normal & 22,012 (71.6) \\
\hline \multicolumn{2}{|l|}{ GGT } \\
\hline$<1.5 \mathrm{~N}$ & $1944(6.3)$ \\
\hline$\geq 1.5 \mathrm{~N}$ & $998(3.2)$ \\
\hline Normal & 21,838 (71.0) \\
\hline \multicolumn{2}{|l|}{ Prothrombin time } \\
\hline Deviation & $1,152(3.7)$ \\
\hline Normal & 23,201 (75.4) \\
\hline \multicolumn{2}{|l|}{ Fasting blood sugar } \\
\hline Deviation & 2,569 (8.4) \\
\hline Normal & 22,836 (74.3) \\
\hline \multicolumn{2}{|l|}{ Cholesterol } \\
\hline Deviation & $8,171(26.6)$ \\
\hline Normal & $17,272(56.2)$ \\
\hline \multicolumn{2}{|l|}{ Triglycerides } \\
\hline Deviation & $5,647(18.4)$ \\
\hline Normal & $18,606(60.5)$ \\
\hline \multicolumn{2}{|l|}{ Thrombocytes } \\
\hline Deviation $(>140000)$ & $346(1.1)$ \\
\hline Deviation $(\leq 140000)$ & $61(0.2)$ \\
\hline Normal & $24,343(79.2)$ \\
\hline \multicolumn{2}{|l|}{ HbsAg antigen } \\
\hline Detected & $552(1.8)$ \\
\hline Not detected & $24,116(78.4)$ \\
\hline \multicolumn{2}{|l|}{ HCV antibodies } \\
\hline Detected & $417(1.4)$ \\
\hline Not detected & 24,164 (78.6) \\
\hline \multicolumn{2}{|c|}{$\begin{array}{l}\text { HBaAg, hepatitis B surface antigen; HCV, hepatitis C virus. } \\
\text { Fasting blood sugar levels were normal in } 74 \% \text { of } \\
\text { subjects, while cholesterol and triglyceride levels were } \\
\text { normal in only } 56-61 \% \text { of subjects. }\end{array}$} \\
\hline
\end{tabular}

\subsection{Prevalence of Hepatic Diseases}

NAFLD was observed in 8,315/30,754 (27.0\%) of subjects in the study population. NAFLD was detected in most of these patients $(8,018 / 8,315 ; 96.4 \%)$ during the course of the study screening, with only 297 (3.6\%) subjects having a diagnosis of NAFLD established before the screening study was initiated.

Steatosis was diagnosed in $80.3 \%$ of those with NAFLD $(6,680 / 8,315)$ and was established in only $3.0 \%$ $(253 / 8,315)$ subjects before the screening study. In the course of screening, steatohepatitis was detected in 16.5\% of NAFLD patients $(1,375 / 8,315)$; according to anamnestic data, steatohepatitis was already established in $0.5 \%$ of subjects before screening $(44 / 8,315)$. In the NAFLD population, the disease at the non-cirrhotic stage was noted in the majority $(8,078 / 8,315 ; 97.1 \%)$ of patients. The disease at the cirrhotic stage was observed in $2.9 \%$ $(237 / 8,315)$ of NAFLD patients.

Alcoholic liver disease (ALD) or alcohol use in dangerous doses (medical history) was noted in 5.2\% of the study population $(1,608 / 30,754)$ and viral hepatitis/virus carrier status was observed in $5.3 \%$ of subjects $(1,617 / 30,754)$.

\subsection{Hepatic Diseases by Age}

The greatest proportion of the NAFLD population was aged 50-59 years (31.1\%), followed by 40-49 years (23.6\%), 60-69 years (18.1\%), 70-79 years (12.2\%), 30-39 years $(8.3 \%)$ then $18-29$ years $(3.5 \%)$.

The age distribution for a range of different hepatic diseases is shown in Table 4. Among patients in the age group 18-29 years, NAFLD was found in 287/5,158 (5.6\%) patients. Of these NAFLD patients aged 18-29 years, steatosis was discovered in $76.7 \%$ patients, steatohepatitis in $21.6 \%$ patients, and cirrhosis in $1.7 \%$ patients.

Similarly, of the NAFLD patients aged 30-39 years, steatosis was discovered in $76.8 \%$ patients, steatohepatitis in $21.7 \%$ patients, and cirrhosis in $1.4 \%$ patients.

Among the patients in the age group 40-49 years, NAFLD was found in 1,960/6,520 (30.1\%) patients. Steatosis was observed in $78.9 \%$ of these NAFLD patients, steatohepatitis in $19.4 \%$ patients, and cirrhosis in $1.7 \%$ patients.

NAFLD was observed in 2,582/6,469 (39.9\%) subjects aged $50-59$ years, primarily steatosis in $80.9 \%$ of these patients, steatohepatitis in $16.8 \%$ and $2.2 \%$ had cirrhosis. In total, $39.4 \%(1,508 / 3,825)$ of patients aged $60-69$ years had NAFLD. NAFLD manifested as steatosis in $81.2 \%$ of these patients and steatohepatitis in $14.7 \%$ patients. The proportion of subjects with cirrhosis (4.1\%) was numerically higher than in the younger age groups.

Among the patients in the age group 70-80 years, NAFLD was found in 1,014/2,693 (37.7\%) of patients. Steatosis was observed in $82.3 \%$ of these NAFLD patients, steatohepatitis in $12.3 \%$ of patients, with cirrhotic disease present in a considerable proportion (5.3\%) of patients.

\subsection{Risk Factors Associated with NAFLD}

Among all patients included in the analysis, risk factors associated with NAFLD were commonly observed (Table 5). Certain risk factors were observed significantly more frequently in subjects in the NAFLD population vs the whole screening population: $69.9 \%$ vs $41.8 \%$ had arterial hypertension, $75.9 \%$ vs $37.6 \%$ had dyslipidemia and $68.8 \%$ vs $33.5 \%$ had hypercholesterolemia (all $\mathrm{P}<0.001$ vs the whole screening population). Regarding insulin resistance, type 2 diabetes was present in $23.1 \%$ of the NAFLD population vs $9.0 \%$ of the whole screening population, while the metabolic syndrome was present in $31.8 \%$ of those with NAFLD vs $12.8 \%$ of the total. In total, $54.2 \%$ of the NAFLD population were obese compared with $35.9 \%$ of the whole study population. 
Table 4. Type of hepatic disease by age

\begin{tabular}{|c|c|c|c|c|}
\hline & \multicolumn{4}{|c|}{ Number of participants (\% of total) [\% of patients with hepatic disease] } \\
\hline \multicolumn{5}{|c|}{ Non-alcoholic fatty liver disease (screening data) } \\
\hline & Non-alcoholic steatosis & Non-alcoholic steatohepatitis & Liver cirrhosis & \\
\hline $18-29$ years & $205(4.0)[25.6]$ & $58(1.1)[7.2]$ & $5(0.1)[0.6]$ & \\
\hline 30-39 years & $518(10.2)[41.7]$ & $148(2.9)$ [11.9] & $10(0.2)[0.8]$ & \\
\hline 40-49 years & $1,500(23.0)[55.2]$ & $374(5.7)[13.8]$ & $34(0.5)$ [1.3] & \\
\hline 50-59 years & $2,013(31.1)[60.5]$ & 429 (6.6) [12.9] & $58(0.9)[1.7]$ & \\
\hline 60-69 years & $1,168(30.5)$ [59.3] & 219 (5.7) [11.1] & $62(1.6)[3.2]$ & \\
\hline 70-80 years & $781(29.0)[64.0]$ & $123(4.6)[10.1]$ & $54(2.0)[4.4]$ & \\
\hline \multicolumn{5}{|c|}{ Alcoholic liver disease } \\
\hline & Alcoholic steatosis & Alcoholic steatohepatitis & & \\
\hline 18-29 years & $4(0.1)[0.5]$ & $6(0.1)[0.7]$ & & \\
\hline 30-39 years & $23(0.5)[1.9]$ & $27(0.5)[2.2]$ & & \\
\hline 40-49 years & $60(0.9)[2.2]$ & $64(1.0)[2.4]$ & & \\
\hline $50-59$ years & $85(1.3)[2.6]$ & $84(1.3)[2.5]$ & & \\
\hline 60-69 years & $58(1.5)[2.9]$ & $40(1.0)[2.0]$ & & \\
\hline $70-80$ years & $23(0.9)[1.9]$ & $15(0.6)[1.2]$ & & \\
\hline \multicolumn{5}{|c|}{ Viral hepatitis/virus carriers } \\
\hline & $\begin{array}{l}\text { Viral } \\
\text { carriers (anamnestic data) }\end{array}$ & Hepatitis B (screening data) & $\begin{array}{lll}\begin{array}{l}\text { Hepatitis } \\
\text { data) }\end{array} & \text { (screening } \\
\end{array}$ & $\begin{array}{l}\text { Combination of hepatitis B and } \\
\text { hepatitis C (screening data) }\end{array}$ \\
\hline 18-29 years & $270(5.2)[33.7]$ & $52(1.0)[6.5]$ & $43(0.8)[5.4]$ & $51(1.0)[6.4]$ \\
\hline 30-39 years & $254(5.0)[20.4]$ & $60(1.2)[4.8]$ & $32(0.6)[2.6]$ & $58(1.1)[4.7]$ \\
\hline 40-49 years & 228 (3.5) 8.4] & $67(1.0)[2.5]$ & $37(0.6)[1.4]$ & $58(0.9)[2.1]$ \\
\hline 50-59 years & $166(2.6)[5.0]$ & $55(0.9)[1.7]$ & $36(0.6)[1.1]$ & $25(0.4)[0.8]$ \\
\hline 60-69 years & $78(2.0)[4.0]$ & $25(0.7)[1.3]$ & $15(0.4)[0.8]$ & $20(0.5)[1.0]$ \\
\hline \multirow[t]{2}{*}{$70-80$ years } & $45(1.7)[3.7]$ & $16(0.6)[1.3]$ & $11(0.4)[0.9]$ & $10(0.4)[0.8]$ \\
\hline & Hemochromatosis & Hepatocellular carcinoma & Autoimmune hepatitis & Drug-induced hepatic injury \\
\hline 18-29 years & $1(0.0)[0.1]$ & $2(0.0)[0.2]$ & $6(0.1)[0.7]$ & $6(0.1)[0.7]$ \\
\hline 30-39 years & $4(0.1)[0.3]$ & $3(0.1)[0.2]$ & $8(0.2)[0.6]$ & $14(0.3)[1.1]$ \\
\hline 40-49 years & $9(0.1)[0.3]$ & $15(0.2)[0.6]$ & $19(0.3)[0.7]$ & $23(0.4)[0.8]$ \\
\hline $50-59$ years & $7(0.1)[0.2]$ & $8(0.1)[0.2]$ & $29(0.4)[0.9]$ & $33(0.5)[1.0]$ \\
\hline 60-69 years & $5(0.1)[0.3]$ & $23(0.6)[1.2]$ & $15(0.4)[0.8]$ & $27(0.7)[1.4]$ \\
\hline $70-80$ years & $5(0.2)[0.4]$ & $18(0.7)[1.5]$ & $10(0.4)[0.8]$ & $12(0.4)$ \\
\hline
\end{tabular}

Table 5. Prevalance of risk factors associated with NAFLD development

\begin{tabular}{|l|l|l|}
\hline & \multicolumn{2}{|l|}{ Number of participants (\%) } \\
\hline Disease & $\begin{array}{l}\text { NAFLD } \\
\text { population } \\
\text { (N=8,315) }\end{array}$ & $\begin{array}{l}\text { Total } \\
\text { population } \\
\text { (N=30,754) }\end{array}$ \\
\hline Abdominal obesity & $4,671(56.2)$ & $11,045(35.9)$ \\
\hline Abdominal obesity, men & $1,464(17.6)$ & $3,075(10.0)$ \\
\hline Abdominal obesity, women & $3,207(38.6)$ & $7,970(25.9)$ \\
\hline Obesity & $4,508(54.2)$ & $7,970(25.9)$ \\
\hline Obesity: stage 1 & $2,750(33.1)$ & $4,827(15.7)$ \\
\hline Obesity: stage 2 & $1,324(15.9)$ & $1,869(6.1)$ \\
\hline Obesity: stage 3 & $434(5.2)$ & $632(2.1)$ \\
\hline Type 1 diabetes & $99(1.2)$ & $154(0.5)$ \\
\hline Type 2 diabetes & $1,922(23.1)$ & $2,755(9.0)$ \\
\hline Hyperglycemia & $1,723(20.7)$ & $2,569(8.4)$ \\
\hline $\begin{array}{l}\text { Women in the age group 45-55 } \\
\text { years }\end{array}$ & $1,596(19.2)$ & $4,019(13.1)$ \\
\hline Arterial hypertension & $5,813(69.9)$ & $12,864(41.8)$ \\
\hline Arterial hypertension, stage 1 & $1,385(16.7)$ & $3,525(11.5)$ \\
\hline Arterial hypertension, stage 2 & $3,416(41.1)$ & $7,372(24.0)$ \\
\hline Arterial hypertension, stage 3 3 & $714(8.6)$ & $1,297(4.2)$ \\
\hline $\begin{array}{l}\text { Cardiovascular system diseases, } \\
\text { except for arterial hypertension }\end{array}$ & $2,321(27.9)$ & $5,445(17.7)$ \\
\hline Menopause & $2,914(35.0)$ & $6,407(20.8)$ \\
\hline Menopause, HRT & $278(3.3)$ & $689(2.2)$ \\
\hline Menopause, no HRT & $2,636(31.7)$ & $5,718(18.6)$ \\
\hline Dyslipidemia & $6,311(75.9)$ & $11,573(37.6)$ \\
\hline Hypertriglyceridemia & $3,908(47.0)$ & $6,482(21.1)$ \\
\hline Lowered level of HDL & $1,478(17.8)$ & $2,445(8.0)$ \\
\hline Hypercholesterolemia & $5,717(68.8)$ & $10,291(33.5)$ \\
\hline Metabolic syndrome & $2,644(31.8)$ & $3,950(12.8)$ \\
\hline HD high-d & &
\end{tabular}

HDL, high-density lipoprotein; HRT, hormone replacement therapy;

NAFLD, non-alcoholic fatty liver disease.
In the two age groups with the highest prevalence of NAFLD, risk factors were particularly frequent. Among patients with NAFLD aged 40-49 years, $71.2 \%$ had dyslipidemia, 65.5\% had hypercholesterolemia and 56.3\% had arterial hypertension. Among the patients with NAFLD aged 50-59 years, $80.6 \%$ had dyslipidemia, $72.6 \%$ had hypercholesterolemia and $75.5 \%$ had arterial hypertension.

\section{Discussion}

The main finding of this large, multicenter prospective population study of more than 30,000 participants was that over one-quarter (27\%) of patients admitted to outpatient clinics in the Russian Federation had ultrasound signs of NAFLD. In $80.3 \%$ of patients, NAFLD presented as steatosis, with NASH observed in $16.8 \%$ of NAFLD cases.

A limited number of studies have assessed the prevalence of NAFLD [Chalasani et al 2012] and these have generally been small studies. The reported NAFLD prevalence varies depending on the population studied and the definition/diagnosis method used. Generally consistent with the findings of the current study, other studies using ultrasound assessment have reported a NAFLD prevalence of $17 \%$ to $46 \%$. [9,15,16] For example, in a US study consisting of 400 middle aged individuals, the prevalence of NAFLD defined by ultrasonography was $46 \%$ and the prevalence of histologically confirmed NASH was $12.2 \%$. [16] In an urban US population $(n=2,287)$, almost one third of the population had hepatic steatosis assessed by magnetic resonance spectroscopy. [10] In a recent study of 
the Chinese population in Hong Kong $(n=2,493)$, the prevalence of NAFLD was $42 \%$ as assessed by ultrasonography and transient elastography [17].

An epidemiological review sought to determine of the current burden of liver disease in Europe. [18] The prevalence rate of NAFLD was found to be $2 \%$ to $44 \%$ in the general European population (including obese children) and $42.6 \%$ to $69.5 \%$ in people with type 2 diabetes. [18] Of note, annual mortality due to cirrhosis ranged from $0.001 \%$ of Greek females to about $0.1 \%$ of Hungarian males. Liver cancer was thought responsible for around 47,000 deaths per year in the European Union.

The current study confirms and extends these findings by demonstrating the high prevalence of NAFLD in a large population from the Russian Federation, where the prevalence of NAFLD was previously unknown. Of particular importance is that $96.4 \%$ of cases of NAFLD were diagnosed during the course of the study screening, highlighting the high proportion of patients who were unaware that they had NAFLD prior to study initiation. In addition to general NAFLD, the current study emphasizes the substantial burden of severe liver disease in this Russian population, with $2.3 \%$ participants exhibiting fibrosis, $0.8 \%$ of participants presenting with cirrhosis and $0.22 \%$ diagnosed with hepatocellular carcinoma.

Patients with NAFLD in the current screening population were most commonly aged between 40 and 59 years. Although not uncommon in young adults, NAFLD occurs more often in the middle aged and the elderly given that the risk factors for its development tend to increase in prevalence with advancing age. [19] NAFLD in the elderly appears to be accompanied by a substantial burden of hepatic (nonalcoholic steatohepatitis, cirrhosis and hepatocellular carcinoma) and extra-hepatic manifestations and complications (cardiovascular disease, extrahepatic neoplasms) than in younger age groups. $[19,20]$ Indeed, in the current study, cirrhotic disease as a proportion of NAFLD cases was observed in a considerable proportion of older participants: $4.1 \%$ of $60-69$ years old and $5.3 \%$ of $70-80$ years old compared with $1.7 \%$ of $18-29$ year olds.

In line with established risk factors, dyslipidemia, hypercholesterolemia, arterial hypertension and obesity were frequently observed in those with NAFLD. However, these risk factors were also present in a large proportion of the whole screening population, which may serve as a warning for future development of NAFLD. Important risk factors for NAFLD progression related to insulin resistance, namely type 2 diabetes and the metabolic syndrome, were present in one-quarter to one-third of those with NAFLD. In addition to liver damage, steatosis can also worsen and/or induce insulin resistance, worsen glycemic control in patients with type 2 diabetes, and predict subsequent development of the metabolic syndrome; NAFLD is also associated with increased cardiovascular risk [1,6,21].

This study may be considered limited in that NAFLD was diagnosed by ultrasonography. Due to the large patient numbers involved, biopsy was not appropriate. However, ultrasonography has proved useful in the diagnosis of NAFLD in other population-based studies $[9,15,22]$ Despite this potential limitation, our results provide important insights into the burden of NAFLD and may have significant clinical importance for NAFLD prevention and management in the Russian Federation and elsewhere. There should be increased awareness of NALFD among clinicians, along with greater consideration for its consequences, associated conditions, causal factors and predictors related to progression.

In conclusion, our results showed that NAFLD is highly prevalent in outpatients in the Russian Federation. There is an urgent need to address the considerable burden of liver disease and the large proportion of individuals with risk factors who may develop NAFLD in the future.

\section{Financial Support}

Editorial assistance was funded by Sanofi.

\section{List of Abbreviations}

NAFLD, non-alcoholic fatty liver disease; NASH, nonalcoholic steatohepatitis; GGT, gamma-glutamyl transpeptidase; ALT, alanine aminotransferase; AST, aspartate aminotransferase; ALD, alcoholic liver disease; $\mathrm{BMI}$, body mass index; HRT, hormone replacement therapy; HBsAg, hepatitis B surface antigen; HCV, hepatitis C virus; ULN, upper limit of normal

\section{References}

[1] Chalasani N, Younossi Z, Lavine JE, Diehl AM, Brunt EM, Cusi $\mathrm{K}$, Charlton M, Sanyal AJ. The diagnosis and management of nonalcoholic fatty liver disease: practice guideline by the American Gastroenterological Association, American Association for the Study of Liver Diseases, and American College of Gastroenterology. Gastroenterology 2012; 142: 1592-1609.

[2] Machado MV, Cortez-Pinto H. Non-invasive diagnosis of nonalcoholic fatty liver disease. A critical appraisal. J Hepatol 2013; 58: 1007-19.

[3] Ratziu V, Bellentani S, Cortez-Pinto H, Day C, Marchesini G. A position statement on NAFLD/NASH based on the EASL 2009 special conference. J Hepatol. 2010; 53: 372-84.

[4] Michelotti GA, Machado MV, Diehl AM. NAFLD, NASH and liver cancer. Nat Rev Gastroenterol Hepatol. 2013;10:656-65.

[5] Musso G, Gambino R, Cassader M, Pagano G. Meta-analysis: natural history of non-alcoholic fatty liver disease (NAFLD) and diagnostic accuracy of non-invasive tests for liver disease severity. Ann Med. 2011; 43: 617-49.

[6] Targher G, Day CP, Bonora E. Risk of cardiovascular disease in patients with nonalcoholic fatty liver disease. N Engl J Med. 2010; 363: 1341-1350.

[7] Ghamar Chehreh ME, Vahedi M, Pourhoseingholi MA, Ashtari S, Khedmat H, Amin M, Zali MR, Alavian SM. Estimation of diagnosis and treatment costs of non-alcoholic Fatty liver disease: a two-year observation. Hepat Mon. 2013; 13: e7382.

[8] Tolman KG, Dalpiaz AS. Treatment of non-alcoholic fatty liver disease. Ther Clin Risk Manag. 2007; 3: 1153-63.

[9] Vernon G, Baranova A, Younossi ZM. Systematic review: the epidemiology and natural history of non-alcoholic fatty liver disease and non-alcoholic steatohepatitis in adults. Aliment Pharmacol Ther. 2011; 34: 274-85.

[10] Browning JD, Szczepaniak LS, Dobbins R, Nuremberg P, Horton JD, Cohen JC, Grundy SM, Hobbs HH. Prevalence of hepatic steatosis in an urban population in the United States: impact of ethnicity. Hepatology. 2004; 40: 1387-95.

[11] Li Z, Xue J, Chen P, Chen L, Yan S, Liu L. Prevalence of nonalcoholic fatty liver disease in mainland of China: a metaanalysis of published studies. J Gastroenterol Hepatol. 2014; 29: 42-51.

[12] Schwenger KJ, Allard JP. Clinical approaches to non-alcoholic fatty liver disease. World J Gastroenterol. 2014; 20: 1712-1723.

[13] Leite NC, Salles GF, Araujo AL, Villela-Nogueira CA, Cardoso CR. Prevalence and associated factors of non-alcoholic fatty liver 
disease in patients with type-2 diabetes mellitus. Liver Int. 2009; 29: 113-9.

[14] Mirrakhimov AE. Nonalcoholic fatty pancreatic disease and cardio-metabolic risk: is there is a place for obstructive sleep apnea? Cardiovasc Diabetol. 2014; 13: 29.

[15] Park SH, Jeon WK, Kim SH, Kim HJ, Park DI, Cho YK, Sung IK, Sohn CI, Keum DK, Kim BI. Prevalence and risk factors of nonalcoholic fatty liver disease among Korean adults. J Gastroenterol Hepatol. 2006; 21: 138-43.

[16] Williams CD, Stengel J, Asike MI, Torres DM, Shaw J, Contreras M, Landt CL, Harrison SA. Prevalence of nonalcoholic fatty liver disease and nonalcoholic steatohepatitis among a largely middleaged population utilizing ultrasound and liver biopsy: a prospective study. Gastroenterology. 2011; 140: 124-31.

[17] Fung J, Lee CK, Chan M, Seto WK, Lai CL, Yuen MF; the Hong Kong Liver Health Census Study Group. High prevalence of nonalcoholic fatty liver disease in the Chinese - results from the Hong Kong liver health census. Liver Int. 2015; 35(2): 542-9.

[18] Blachier M, Leleu H, Peck-Radosavljevic M, Valla DC, RoudotThoraval F. The burden of liver disease in Europe: a review of available epidemiological data. J Hepatol. 2013; 58: 593-608.
[19] Bertolotti M, Lonardo A, Mussi C, Baldelli E, Pellegrini E, Ballestri S, Romagnoli D, Loria P. Nonalcoholic fatty liver disease and aging: Epidemiology to management. World J Gastroenterol. 2014; 20: 14185-14204.

[20] Noureddin M, Yates KP, Vaughn IA, Neuschwander-Tetri BA, Sanyal AJ, McCullough A, Merriman R, Hameed B, Doo E, Kleiner DE, Behling C, Loomba R; NASH CRN. Clinical and histological determinants of nonalcoholic steatohepatitis and advanced fibrosis in elderly patients. Hepatology. 2013; 58: 164454.

[21] Leite NC, Villela-Nogueira CA, Cardoso CR, Salles GF. Nonalcoholic fatty liver disease and diabetes: from physiopathological interplay to diagnosis and treatment. World J Gastroenterol. 2014; 20: 8377-92.

[22] Xu C, Yu C, Ma H, Xu L, Miao M, Li Y. Prevalence and risk factors for the development of nonalcoholic fatty liver disease in a nonobese Chinese population: the Zhejiang Zhenhai Study. Am J Gastroenterol. 2013; 108: 1299-304. 